\title{
Neurocutaneous Melanocytosis: Cytological, Clinical and Radiological Assessment in a Patient Presented with Congenital Melanocytic Nevi and Seizure Disorder: Case Report and Review of the Literature
}

\author{
Ahmad M Daqour ${ }^{1 *}$, Motee N Ashhab ${ }^{2 *}$, Ayman A Salman ${ }^{1}$ and Izzeddin A Bakri ${ }^{3}$ \\ ${ }^{1}$ Department of Neurosurgery, Al-Makassed Islamic Charitable Hospital, Palestine \\ ${ }^{2}$ Department of Pediatrics, Al-Makassed Islamic Charitable Hospital, Palestine \\ ${ }^{3}$ Department of Clinical Pathology, Al-Makassed Islamic Charitable Hospital, Palestine
}

\begin{abstract}
Neurocutaneous Melanocytosis (NCM) is a rare congenital disorder characterized by multiple melanocytic nevi scattered all over the body, in addition to leptomeninges. It is suggested that NCM is developed due to abnormal proliferation of melanin-producing cells following dysmorphogenesis in the embryology life. The clinical symptoms of NCM depend on the size and site of the lesion. One of the most common sites is the anterior temporal lobe. Management options are limited in such patient and the prognosis is generally poor. Clinical, radiological, along with CSF cytology can establish the diagnosis of NCM. Surgical intervention carries temporary better results than adjuvant therapy in patients with NCM.
\end{abstract}

Keywords

NCM, Leptomeninges, Dysmorphogenesis, Temporal lobe, Atypical melanocytes

\section{Introduction}

Neurocutaneous Melanocytosis (NCM) is an uncommon fatal congenital disorder described by the presence of large melanocytic nevi along with benign or malignant melanocytic proliferation of the leptomeninges [1-3]. Although the exact pathogenesis of NCM is not entirely perceived, it is believed to result from dysregulation and hence impaired production of the neural crest during embryogenesis [4] which eventually leads to anomalous multiplication of melanin-producing cells in the skin and leptomeninges $[5,6]$. Variety of brain areas are found to be implicated in NCM which include: Dentate nucleus of the cerebellum, amygdala, and Virchow-Robin peri-vascular spaces [3,7-15].

Fewer than 200 cases of NCM have been reported in the literature [16], since the first time it was described by Rokitansky in $1861[16,17]$. Most cases of NCM are sporadic and tend to occur in males and females with a ratio of $1: 1$ with no gender predilection [18-20].

Since NCM is not extensively studied with some pathophysiological aspects are still hidden, there is a humble experience using the adjuvant therapy. In those patients where chemotherapy and/or radiotherapy were used, the prognosis was not changed, and death was the fate of all patients [21].
We report an 11 -month- old female patient, who presented to our unit with congenital hyper-melanocytic nevi, with an evolving seizure disorder. The diagnosis of NCM was applied by correlating the clinical picture with MRI findings along with cerebrospinal fluid (CSF) cytology result.

\section{Case Report}

We report an 11 -month- old female patient, a product of Normal Spontaneous Vaginal Delivery (NSVD), full term, with

*Corresponding authors: Ahmad M Daqour, Department of Neurosurgery, Level 1, Al-Makassed Islamic Charitable Hospital, Palestine, Tel: +970546016522

Motee N Ashhab, Department of Pediatrics, Level 1, Al-Makassed Islamic Charitable Hospital, Palestine, Tel: +970548007449

Accepted: August 07, 2021

Published online: August 09, 2021

Citation: Daqour AM, Ashhab MN, Salman AA, et al. (2021) Neurocutaneous Melanocytosis: Cytological, Clinical and Radiological Assessment in a Patient Presented with Congenital Melanocytic Nevi and Seizure Disorder: Case Report and Review of the Literature. J Neurosurg Res Rev 4(1):61-65 
Citation: Daqour AM, Ashhab MN, Salman AA, et al. (2021) Neurocutaneous Melanocytosis: Cytological, Clinical and Radiological Assessment in a Patient Presented with Congenital Melanocytic Nevi and Seizure Disorder: Case Report and Review of the Literature. J Neurosurg Res Rev 4(1):61-65

uneventful antenatal and perinatal history, and birth weight of $2 \mathrm{~kg}$. She is developmentally up-to-date, started walking recently, says few monosyllable words, plays with toys and Lego, and interact with other children. She is taking a regular diet for age with vaccination up-to-date.

Parents are not consanguineous, the patient has 2 female siblings (3-years and 5-years), healthy, with no history of similar cases in extended family and no history of other neuro-cutaneous diseases.

The patient was found since birth to have large nevi covering all parts of her body, in different sizes. She was following up with a dermatologist since birth. The skin lesions were noticed to be increasing in number and size as the child is growing.

At age of 4 months, the patient developed her first seizure in form of atony and cyanosis. The family sought a medical advice and whole CNS MRI was done at age of 4 months which revealed normal brain parenchyma, no obvious intracranial lesions, normal ventricular system, and no spinal lesions. In addition, complete septic workup including: $\mathrm{CBC}, \mathrm{CRP}$, blood culture, urine culture, CSF analysis and culture was done and showed negative infectious findings.

Since then, the patient was maintained on phenobarbital dose / $\mathrm{kg}$ with partial control on her seizures. The seizure is described by family as generalized abnormal movements with lip smacking and staring, for less than a minute each time. However, there is no history of status epilepticus. For this reason, levetiracetam does / $\mathrm{kg}$ was added.

Control Brain MRI at the age of 10 months has shown a T2 hypo-intense signal (Figure $1 \mathrm{~A}$ ), and $\mathrm{T} 1$ hyper-intense signal (Figure 1B) at the left temporal peri-sylvian area with dilatation of the lateral and 3rd ventricles. No other intraparenchymal lesions have been identified, no cerebellar lesions, and no intracranial hemorrhage. Moreover, whole spine MRI showed normal alignment of the vertebrae, with no apparent lesions involving the vertebral bodies, and no compression on the spinal cord at any given level.

Abdominal ultrasound was regularly done since birth, the last one was performed at the age of 10 months and showed "mild right hydro-nephrosis, and $8.84 \mathrm{~mm}$ hyperechoic lesion in the liver, not resembling hemangioma, and could be calcification". Echocardiography showed a small Patent Foramen Ovale (PFO). Ophthalmic exam was also done at the age of 10 months and showed absence of choroid pigmentation.

Moreover, Electroencephalography (EEG) was done at age of 11 months and showed diffuse mixture of theta activity and scattered delta wavers, runs of left-sided central temporal spikes and sharp waves of epileptic nature.

On physical exam, the patient is well, playful, with no respiratory distress, or dehydration. She is not jaundiced or cyanosed. No cleft palate or lip. There is a nevus on the hard palate. There are multiple large dark hairy nevi over all parts of body (Figure 2A), in different sizes, largest involving the whole right leg (Figure $2 \mathrm{~B}$ ) with small hyperemic lesion on the back, and a hypopigmented irregular shaped lesion on the left trunk. Neurological exam revealed a conscious and active girl, intact cranial nerves grossly, pupils are both reactive to light and accommodation, she has normal sensations, and moving all limbs freely, with a normal tone and power, and deep tendon reflexes are +2 . It is difficult to assess the gait as she is not walking independently yet. Chest exam revealed good air entry bilaterally. No murmur appreciated. The abdomen was soft, lax, and not tender. The liver is palpable $2 \mathrm{~cm}$ below the costal margin and the spleen just palpable. No palpable lymph nodes.

The patient has normal female genitalia. Normal social interaction and eyes following. There is no history of chocking or recurrent infections.

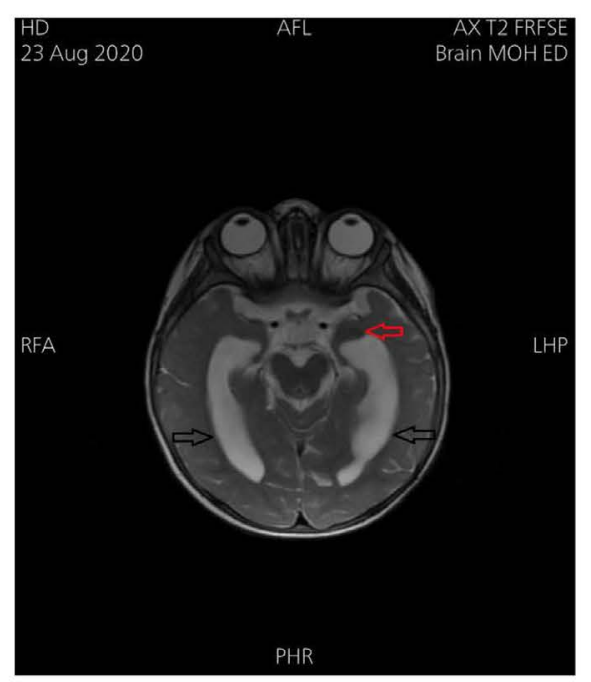

$1 \mathrm{~A}$

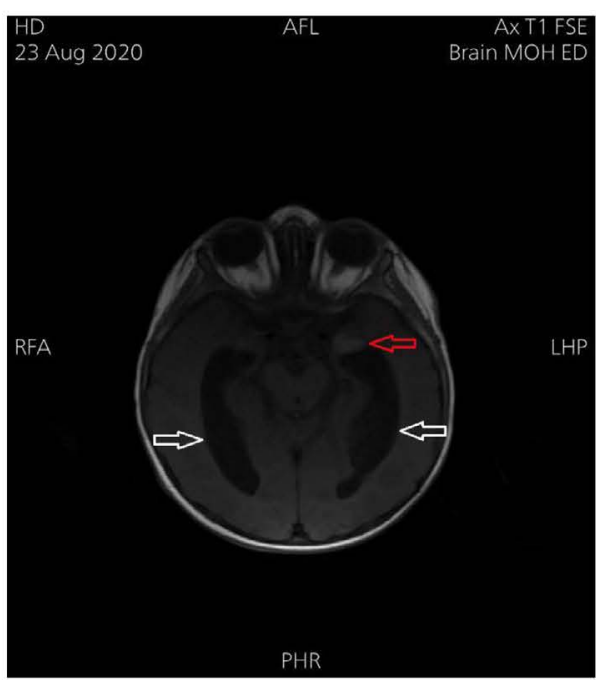

$1 \mathrm{~B}$

Figure 1: A) Axial T2-weighted MRI sequence without contrast showing a hypointense signal at the left temporal peri-sylvian area (Red arrow). Also, marked dilatation of the lateral ventricles is found (Black arrows); B) Axial T1-weighted MRI sequence without contrast showing a hyperintense signal at the left temporal peri-sylvian area (Red arrow). Note marked dilatation of the lateral ventricles as well (White arrows). In both sequences, no other lesions have been identified either in the cerebral cortex or brain parenchyma 


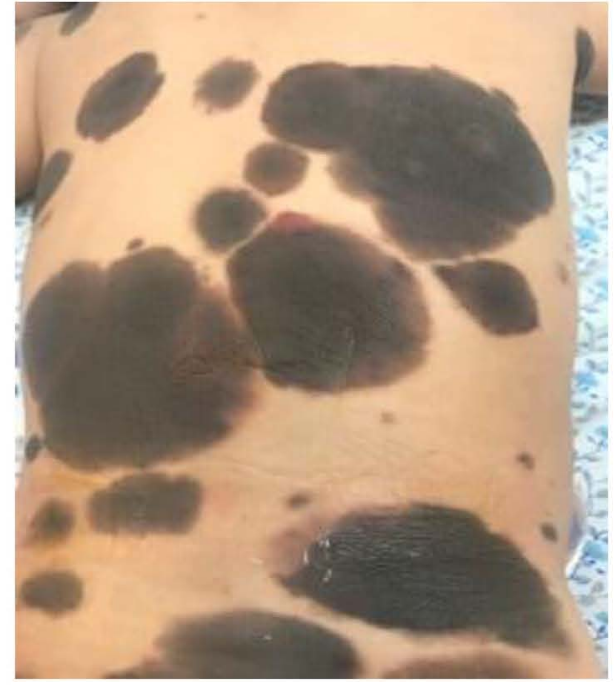

$2 \mathrm{~A}$

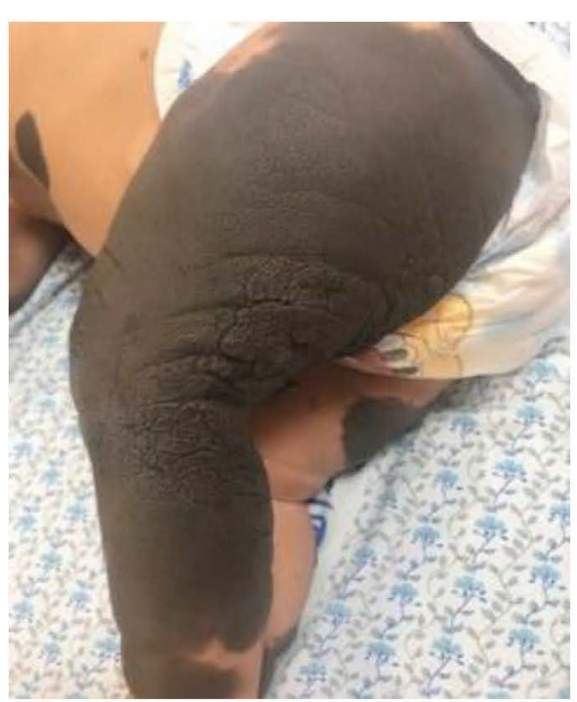

$2 \mathrm{~B}$

Figure 2: A) Multiple, different-sized, melanocytic nevi scattered over the back and buttocks; B) Large, melanocytic nevus over the whole right lower limb.

Pertinent laboratory values included Complete Blood Count (CBC), International Normalized Ratio (INR), Prothrombin Time (PT), Partial Thromboplastin Time (PTT), Liver Function Tests (AST, ALT, ALK), Serum Electrolytes, Urinalysis, and Kidney Function Tests (Creatinine and Blood Urea Nitrogen) and were within normal ranges.

Then, under fully sterile technique, Lumbar Puncture (LP) was performed, and the opening pressure was measured and found low: $5 \mathrm{~cm} \mathrm{H}_{2} \mathrm{O}$. A sample of CSF sent for analysis and culture, with an additional sample sent for cytology analysis. CSF analysis showed: Total cells: 200, WBC: 0, Glucose: 43, Protein: 47, Random Blood Sugar (RBS): 81. CSF culture showed no growth. The CSF cytology revealed "Atypical individual cells and occasional small clusters of almost uniform round mononuclear cells with pseudopod-like periphery". These features are consistent with Neurocutaneous Melanocytosis (NCM) in correlation with clinical and imaging findings.

\section{Discussion}

Neurocutaneous Melanocytosis (NCM) is considered a rare, but lethal, congenital melanocytic disorder [1-3]. It was first described by Rokitansky in 1861 [16,17], and less than 200 cases have been reported in the literature since that time [16]. The exact etiology of this rare entity remains uncertain although some authors suggested dysregulation of the neural crest during the embryology life [4]. In addition, disintegration in hepatocyte growth factor seems to have a considerable role in the pathogenesis of NCM [6], due to its role in the proliferation of melanocytes. Most cases of NCM are sporadic, some authors noted for the NCM to be higher in Caucasian people, with no sex predilection $[18,19]$.

High-volume work has been published while studying the neuropathology of NCM. Rokitansky, et al. [17] firstly described multiple melanocytic pigmentations in the ventricular ependymal lining. Extensive work followed that and melanocytic infiltrations have been found in the cerebellum, specifically the dentate nucleus, and the anterior temporal lobes, especially the amygdala [3,7-14]. Furthermore, some reports found melanocytic infiltrations in the Virchow-Robin perivascular spaces $[22,23]$. It is worthy noting that unlike the periphery of the body, the majority of melanocytes infiltrating the leptomeninges and CNS in neurocutaneous melanocytosis are unpigmented [13]. Less frequently, NCM may be demonstrated after death $[22,23]$.

The presenting symptoms in patient with NCM vary according to lesion size, site, and presence of hydrocephalus, and include: generalized seizures (48\%); as in our case, vomiting (42\%), headache (35\%), cranial nerve palsies (26\%), large head circumference (23\%), myelopathy (10\%), and meningeal signs $(3 \%)[3,20,24,25]$. One of the commonest sites for melanocytic infiltration is in the anterior temporal lobe in the region of the amygdala, which can provoke seizure disorder $[3,24,25]$, as illustrated in our reported case. CNS tumors in NCM are usually highly malignant [1] and malignant transformation of leptomeningeal melanocytosis to leptomeningeal melanoma has been reported in $40 \%$ to $60 \%$ of symptomatic cases [6]. Neurologic manifestations generally occur before the age of 2-years in the vast majority of patients [26] with approximately $50 \%$ of patients with CNS involvement already manifest in the first year of life [27]. Less frequently, patients may present with signs or symptoms of NCM in the $2^{\text {nd }}$ or $3^{\text {rd }}$ decade of life [28].

Most of the cases reported in the literature have shown a rapid functional impairment along with decreased chance in life. A significant number of patients died in less than 3-years since they presented with neurologic features [26]. This could be explained, to some extent, by development of atypical melanocytes from the covering layers of the brain into the different eloquent areas of the brain which can then lead to hydrocephalus, high intracranial pressure, and seizures $[6,20]$.

CSF analysis and cytology can help in diagnosing patients suspected to have NCM. CSF analysis usually demonstrate 
Citation: Daqour AM, Ashhab MN, Salman AA, et al. (2021) Neurocutaneous Melanocytosis: Cytological, Clinical and Radiological Assessment in a Patient Presented with Congenital Melanocytic Nevi and Seizure Disorder: Case Report and Review of the Literature. J Neurosurg Res Rev 4(1):61-65

high levels of protein and White Blood Cells (WBCs) accompanied by normal values of glucose. The presence of atypical melanocytes in the CSF could point into the diagnosis of NCM supported by the clinical and radiological findings. Imaging studies, such as: Computed Tomography (CT) [29] and Magnetic Resonance Image (MRI) [30] are frequently used to identify intra-cranial space-occupying lesions and/ or presence of hydrocephalus with variable degrees of sensitivity.

In addition, Electroencephalography (EEG) can be utilized to illustrate the electrical activity in patient with a neurological element of NCM. In patients presenting with a seizure disorder, as in our work, the EEG usually demonstrates central and/or temporal spikes along with diffuse mixture of theta activity and delta waves.

The definitive treatment for NCM is still controversial. Using chemotherapy or radiotherapy has not shown promising results with most patients who received adjuvant therapy passed away shortly after beginning of the treatment [21]. On the other hand, patients who underwent neurosurgical intervention in the form of Ventriculo-Peritoneal (VP) shunt insertion when they had hydrocephalus had improvement for a short period of time [26,31]. However, one of the feared complications following VP shunt insertion in patients with NCM is peritoneal metastasis of melanoma [13,26,31-36]. In those patients who are suffering from drug-resistant seizure disorder with imaging evidence of melanocytic infiltration of the amygdala, then neurosurgical intervention in the form of anterior temporal lobectomy and hippocampectomy could control seizures $[12,13]$. However, our patient was transferred to another highly specialized center to get a chance of chemotherapy and radiotherapy as these modalities of treatment are not available at our hospital.

Social and psychological support is recommended for the families of these patients as the outcome is usually poor and early death is the terminal fate in almost all patients.

\section{Funding}

Nil.

\section{Conflict of Interest}

None declared.

\section{References}

1. Arunkumar MJ, Ranjan A, Jacob M, et al. (2001) Neurocutaneous melanosis: A case of primary intracranial melanoma with metastasis. ClinOncol (R Coll Radiol) 13: 52-54.

2. Lovett Lovett A, Maari C, Decarie JC, et al. (2009) Large congenita melanocytic nevi and neurocutaneous melanocytosis: One pediatric center's experience. J Am Acad Dermatol 61: 766-774.

3. Ye BS, Cho YJ, Jang SH, et al. (2008) Neurocutaneous melanosis presenting as chronic partial epilepsy. J Clin Neurol 4: 134-137.

4. Bolande RP (1997) Neurocristopathy: Its growth and development in 20 years. Pediatr Pathol Lab Med 17:1-25.

5. Burstein F, Seier H, Hudgins PA, et al. (2005) Neurocutaneous melanosis. J Craniofac Surg 16:874-876.
6. Acosta FLJr, Binder DK, Barkovich AJ, etal. (2005) Neurocutaneous melanosis presenting with hydrocephalus. Case report and review of the literature. J Neurosurg 102:96-100.

7. Frieden I, Williams M, Barkovich AJ (1994) Giant congenital melanocytic nevi: Brain magnetic resonance findings in neurologically asymptomatic children. J Am Acad Dematol 31: 423-429.

8. Demirci A, Kawamura Y, SzeGet, et al. (1995) MR of parenchymal neurocutaneous melanosis. AJNR Am J Neuroradiol 16: 603-606.

9. Foster RD, Williams ML, Barkovich AJ, et al. (2001) Giant congenital melanocytic nevi: The significance of neurocutaneous melanosis in neurologically asymptomatic children. Plast Reconstr Surg 107: 933-941.

10. Edelstein S, Naidich TP, Newton TH (2005) The rare phakomatoses. In: P Tortori-Donati, Pediatric Neuroradiology. Brain, Springer, Berlin and Heidelberg: 819-854.

11. Jo'zwiak S, Borkowska J (2008) Neurocutaneous melanosis. In: M Ruggieri, I Pascual-Castroviejo, C Di Rocco (edn), Neurocutaneous Disorders. Springer-Verlag, Vienna, New York, 1-17.

12. Fu YJ, Morota N, Nakagawa A, et al. (2010) Neurocutaneous melanosis: Surgical pathological features of an apparently hamartomatous lesion in the amygdala. J Neurosurg Pediatr 6: 82-86.

13. Flores-Sarnat L (2013) Neurocutaneous melanocytosis. Handb Clin Neurol 111: 369-388.

14. Fox H, Emery JL, Goodbody RA, et al. (1964) Neurocutaneous melanosis. Arch Dis Child 39: 508-516.

15. Salisbury JR, Rose PE (1989) Primary central nervous malignant melanoma in the bathing trunk naevus syndrome. Postgrad Med J 65: 387-389.

16. Barkovich AJ (2005) Pediatric neuroimaging. Philadelphia, Pa: Lippincott Williams \& Wilkins 487-488.

17. Rokitansky J (1861) Ein ausgezeineter fall von PigmentMal mit ausgebreiteter Pigmentirung der inneren Hirn und R€uckenmarksha"ute. Allg Wien Med Ztg 6: 113-116.

18. Poe LB, Roitberg D, Galyon DD (1994) Neurocutaneous melanosis presenting as an intradural mass of the cervical canal: Magnetic resonance features and the presence of melanin as a clue to diagnosis-case report. Neurosurgery 35: 741-743.

19. Kalayci M, Cagavi F, Bayar U, et al. (2006) Neurocutaneous melanosis associated with Dandy-Walker malformation. Acta Neurochir (Wien) 148: 1103-1106.

20. Pavlidou E, Hagel C, Papavasilliou A, et al. (2008) Neurocutaneous melanosis: Report of three cases and up-to-date review. J Child Neurol 23: 1382-1391.

21. Di Rocco F, Sabatino G, Koutzoglou M, et al. (2003) Neurocutaneous melanosis. Child Nervous Syst 20: 23-28.

22. Slaughter JC, Hardman JM, Kempe LG, et al. (1969) Neurocutaneous melanosis and leptomeningeal melanomatosis in children. Arch Pathol 88: 298-304.

23. Pascual-Castroviejo I (1987) Neurocutaneous melanosis. In: M Gomez (edn), Neurocutaneous Diseases. Butterworth, Boston, 329-334.

24. Kim YJ, Won YD, Kim KT, et al. (2006) Parenchymal neurocutaneous melanosis in association with intraventricular dermoid and Dandy-Walker variant: A case report. Korean J Radiol 7: 145-148. 
Citation: Daqour AM, Ashhab MN, Salman AA, et al. (2021) Neurocutaneous Melanocytosis: Cytological, Clinical and Radiological Assessment in a Patient Presented with Congenital Melanocytic Nevi and Seizure Disorder: Case Report and Review of the Literature. J Neurosurg Res Rev 4(1):61-65

25. Azzoni A, Argentieri R, Raja M (2001) Neurocutaneous melanosis and psychosis: A case report. Psychiatry Clin Neurosci 55: 93-95.

26. Kadonaga JN, Frieden IJ (1991) Neurocutaneous melanosis. Definition and review of the literature. J Am Acad Dermatol 24:747-755.

27. Mena-Cedillos CA, Valencia-Herrera AM, Arroyo- Pineda Al, et al. (2002) Neurocutaneous melanosis in association with the Dandy-Walker complex, complicated by melanoma: Report of a case and literature review. Pediatric Dermatology 19: 237-242.

28. Schaffer JV, McNiff JM, Bolognia JL (2001) Cerebral mass due to neurocutaneous melanosis: Eight years later. Pediatr Dermatol 18: 369-377.

29. Enzman DR, Krikorian J, Yorke C, et al. (1978) Computed tomography in leptomeningeal spread of tumor. J Comput Assist Tomogr 2: 448-455.

30. Britton J, March H, Kendall B, et al. (1988) MRI and hydrocephalus in childhood. Neuroradiology 30:310-314.

31. Humes RA, Roskamp J, Eisenbrey AB (1984) Melanosis and hydrocephalus: Report of four cases. J Neurosurg 61: 365-368.
32. Faillace WJ, Okawara SH, McDonald JV (1984) Neurocutaneous melanosis with extensive intracerebral and spinal cord involvement: Report of two cases. J Neurosurg 61: 782-785.

33. Gattuso P, Carson HJ, Attal H, et al. (1995) Peritoneal implantation of meningeal melanosis via ventriculoperitoneal shunt: A case report and review of the literature. Diagn Cytopathol 13: 257259.

34. Shinno K, Nagashiro S, UnoM (2003) Neurocutaneous melanosis associated with malignant leptomeningeal melanoma in an adult: clinical significance of 5-S-cysteinyldopa in the cerebrospinal fluid: Case report. Neurol Med Chir (Tokyo) 43:619-625.

35. Hsueh Ch, Ho Ch, Chiu N, et al. (2004) Neurocutaneous melanosis with hydrocephalus: Report of one case. Acta Neurol Taiwan 13: 29-33.

36. Subbiah V, Wolff JEA (2010) Rapid response to therapy of neurocutaneous melanosis with leptomeningeal melanoma. Pediatr Blood Cancer 54: 180-181. 